\title{
Dificultades para la Transversalización de la Perspectiva de Género en una Institución de Educación Superior
}

\section{Difficulties to Gender Mainstreaming at a Higher Education Institution}

\author{
Alejandra Montes-de-Oca-O’Reilly \\ Universidad Autónoma del Estado de Morelos, México
}

\begin{abstract}
Investigaciones previas muestran que existen resistencias para la transversalización de la perspectiva de género en las Instituciones de Educación Superior (IES). Este estudio de carácter cualitativo tuvo por objetivo conocer las representaciones que tienen siete mujeres y siete hombres directivos $/$ as $^{1}$ académicos de una universidad pública $^{2}$ de México sobre la perspectiva de género, así como sus disposiciones para transversalizar, o en su caso, incorporar el enfoque de género en su institución. Bajo un diseño de estudio de caso, se aplicaron entrevistas semiestructuradas y se encontró que la mayoría de los/as directivos/as conoce poco sobre la perspectiva de género y ninguno/a tiene una visión histórica de qué grupos han venido impulsando políticas públicas sobre equidad de género. Si bien la política pública indica que debe incorporarse la perspectiva de género, algunos/as participantes informaron que en la práctica dentro de la institución existen resistencias por parte de autoridades, investigadores/as y docentes. Se observó escaso interés por transversalizar la perspectiva de género en el curriculum. Los hallazgos sugieren que los programas de transversalización de la perspectiva de género proporcionen una visión histórica de los avances logrados en políticas públicas de equidad y cuáles son exactamente los planteamientos de las diversas posturas académicas sobre el tema.
\end{abstract}

Descriptores: Género; Educación superior; México; Transversalización; Políticas educativas.

Previous research shows that there are resistances to gender-mainstreaming in Higher Education Institutions. This qualitative study examines representations of the gender perspective of seven women and seven men authorities at a public university in Mexico, as well as their dispositions to mainstream, or incorporate the gender perspective in their institution. This is a case study in which, through semistructured interviews, it was found that most of them have poor knowledge of the gender perspective and none has a historical understanding of which groups have been promoting public policies on gender equity. Although public policy indicates that the gender perspective must be present, some participants reported that in practice there are resistances on the part of authorities, researchers and teachers within the institution. There was little interest in gender mainstreaming in the curriculum. As a result, it is recommended that gender-mainstreaming programs provide a historical overview of the progress made in equity policies as well as the different academic approaches on the subject.

Keywords: Gender; Higher education; Mexico; Mainstreaming; Education policies.

\footnotetext{
${ }^{1}$ En congruencia con el enfoque de género de este trabajo, hago uso de un lenguaje incluyente mediante el uso de la diagonal pues parto del supuesto de que las transformaciones en el lenguaje dan lugar a transformaciones en las prácticas. Cuando hago referencia a autores/as que utilizan el masculino como genérico, lo hago como está escrito en sus propios textos.

${ }^{2}$ La universidad en donde se realizó esta investigación se encuentra localizada en la zona de México conocida como "El Bajío", en el centro del País. Por razones de confidencialidad, se han omitido datos que pudieran llevar a la identificación de las personas entrevistadas.
}

*Contacto: alemoreilly@gmail.com

Recibido: $\quad 10 / 08 / 2014$

ISSN: 07 18-7378

$1^{\text {a }}$ Evaluación: 25/02/2015

www.rinace.net/rlei/

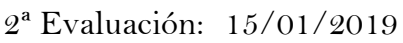

Aceptado: 30/03/2019 


\section{Introducción}

La colaboración durante décadas entre académicas/os del género, activistas e integrantes del aparato gubernamental ha logrado avances en el tema de equidad de género tanto en la sociedad como en la educación superior. Sin embargo, la realidad es que aún falta mucho por avanzar. Las/os activistas han logrado colocar en el debate público el tema de la violencia de género en sus variantes diversas: feminicidios ${ }^{3}$, violencia hacia las mujeres por parte de sus parejas, violencia hacia las mujeres en las calles (violación, manoseo, abuso verbal), prostitución forzosa, así como el hostigamiento y el abuso sexual, entre otros.

Además de la violencia de género, existen todavía paradigmas sociales androcéntricos con los que se sigue reproduciendo lo que Bourdieu (2000) llamó "la dominación masculina". En la esfera pública, por ejemplo, la falta de una auténtica democracia en donde la población esté representada de manera proporcional conlleva una baja representación de las mujeres en espacios públicos y de toma de decisión. Por consiguiente, las políticas públicas y la distribución de recursos tienen un sesgo de género ${ }^{4}$. La doble jornada que tienen las mujeres que trabajan fuera del hogar, la diferencia salarial entre los sexos, la feminización de la pobreza, etc. son otros de los muchos asuntos pendientes en el tema de la equidad.

El ámbito de la educación superior no escapa de esta realidad social. Las instituciones educativas transmiten códigos de género que reproducen la jerarquía varones-mujeres (Acker, 1994). Algunas investigaciones demuestran que el mero acceso de las mujeres a la educación formal no garantiza que las inequidades de género se reduzcan de manera inmediata (Bustos, 2008; García, 2004, 2005; Morley, 1994; Vázquez y Zapata, 2005). Problemas como acoso sexual (Castro y Vázquez, 2008; Sánchez, 2008), la poca o nula representación de las académicas en órganos de toma de decisión (Buquet et al., 2006; PUEG, 2010), el limitado acceso de las académicas a redes informales de conocimiento la falta de políticas de equidad de género en la distribución de recursos, los obstáculos informales para el ingreso y permanencia de las mujeres, la ocupación de espacios según el sexo, los sistemas de evaluación de docentes y de investigadores/as basados en criterios androcéntricos que no consideran tiempo de maternidad, la inequidad de género en el uso de tecnologías (Yurén et al., 2013) por ejemplo, entre otros, son algunos de los retos que enfrentan las instituciones de educación superior (IES) en materia de equidad de género.

Si se examina la realidad desde la óptica de género, se observan inequidades substanciales en las universidades. Por ejemplo, en México existe el Sistema Nacional de Investigadores (SNI), que es un mecanismo de un organismo gubernamental, el Consejo Nacional de Ciencia y Tecnología (CONACYT) para, entre otras aplicaciones, evaluar el desempeño de los/as investigadores/as. En 2016, 15,992 hombres y 9,080 mujeres pertenecían a este Sistema (Rodríguez, 2016). Es decir, en ese año las mujeres conformaban el $36.21 \%$ del padrón del SNI. Mientras que el $63.78 \%$ lo constituían los hombres. La diferencia es casi de 28 puntos porcentuales. Asimismo, los niveles más altos en el SNI (Nivel II, III y Eméritos) son ocupados en su mayoría por hombres (CONACYT, 2019). Una explicación

\footnotetext{
` Como resultado de la reflexión de las académicas del género, se ha diferenciado entre los términos "femicidio" (que se refiere al asesinato de una mujer) y "feminicidio" (que significa "asesinato de una mujer a manos de un hombre por machismo o misoginia” según la Real Academia Española.

${ }^{4}$ Esto no implica que la simple inclusión de las mujeres provoque cambios a favor de la equidad de género, pues muchas mujeres no tienen conciencia de las inequidades.
} 
desde el enfoque de género es que existe un techo de cristal (glass ceiling). El techo de cristal es una metáfora que surge desde la perspectiva de género para denominar a aquellas barreras que no se ven, pero que existen y que impiden que las mujeres ocupen los escalafones más altos dentro de las jerarquías institucionales. Ejemplos como estos son útiles para pensarlos en tanto problemas que afectan mayormente a las mujeres como un colectivo y no como temas que cada mujer debe aprender a resolver de manera individual. El reconocimiento social paulatino de ese tipo de fenómenos va "pavimentando el camino" para la formulación de políticas públicas que los atiendan.

Así como las inequidades de género afectan la vida de mujeres y hombres, también influyen en la producción del conocimiento que se genera en las IES. Es que el género, al ser transversal, también atraviesa estructuras como las IES, y a su vez, tanto el contenido como la dirección del conocimiento. La vasta literatura sobre epistemología con perspectiva de género muestra cómo el género influye en la forma y rumbo de la ciencia (Anderson, 2017; Harding y Norberg, 2005; Longino, 1993; Rose, 1983). Se ha mostrado cómo en la investigación científica existe una división del trabajo teórico basada en el género, así como un simbolismo de género en la jerarquía del conocimiento y en el contenido de las teorías (Anderson, 1995; Rosser, 1992). Así, la perspectiva de género constituye una de las contribuciones más importantes de las humanidades y las ciencias sociales al resto de los campos del conocimiento.

Sin embargo, muchas veces la perspectiva de género es conocida únicamente por algunos/as académicos/as de las ciencias sociales, las humanidades o las ciencias del comportamiento. Por ello, para la solución a la problemática de la inequidad de género en las IES se requiere de una transversalización de la perspectiva de género y que ésta permee ampliamente en todos los ámbitos de la educación superior. Entre otros aspectos, ello implica que los/as agentes educativos/as y los/as tomadores/as de decisiones incidan desde la perspectiva de género tanto en el curriculum explícito como en el curriculum oculto. Por ello, se considera necesario que las/os tomadores/as de decisiones académicas que tienen que ver con el curriculum y la formación de universitarios/as conozcan algunos fundamentos básicos de la Teoría de Género, como el que mujeres y hombres no tienen esencias derivadas de la biología, sino que son construcciones simbólicas concernientes al orden del lenguaje y de las representaciones (Lamas, 2000) y que tanto en las sociedades como en el ámbito académico persiste una ideología androcéntrica. En este sentido, resulta útil la definición de Scott (1986, p. 1076) "el género es un elemento constitutivo de las relaciones sociales basadas en las diferencias que distinguen los sexos y el género es una forma primaria de relaciones significantes de poder".

En este escenario, el estudio tuvo por objetivo conocer las representaciones que tienen las/os directivas/os académicos/as en una institución de educación superior mexicana sobre la perspectiva de género, así como sus disposiciones para transversalizar, o en su caso, incorporar el enfoque de género en dicha institución.

\section{Transversalización de la perspectiva de género}

Los grupos de mujeres organizadas han impulsado reformas en ámbitos diversos que han transformado las vidas de millones de mujeres en el planeta. Las organizaciones de mujeres y los movimientos trasnacionales de mujeres han logrado incidir para que organismos como la Organización de las Naciones Unidas promuevan la equidad de 
género en todo el orbe (Otto, 2010; West, 1999). Ejemplo de ello es la integración de la perspectiva de género en las conferencias de mujeres de México (1975), Bruselas (1976), Bangkok y Nueva York (1980), Nairobi (1985), Beijing (1995), entre otras. Durante la IV Conferencia Mundial sobre la Mujer que tuvo lugar en Beijing en 1995, los gobiernos participantes y las agencias de la ONU asumieron el compromiso de promover la transversalidad del enfoque de género como la estrategia más adecuada para promover los derechos de las mujeres (ONU, 1995; PNUD Colombia, 2005).

Los lineamientos globales han ido permeando lentamente en las políticas públicas de México. En los Planes Nacionales de Desarrollo (PND) (2001-2006) y (2007-2012) se señala la necesidad de transversalizar la perspectiva de género. En el primero se menciona una sola vez cuando se apunta a la: "transversalidad en las políticas públicas con perspectiva de género” (Gobierno de la República, 2001, p. 47) como un criterio a seguir por el Instituto Nacional de las Mujeres (InMujeres). En el segundo se asegura que "se incorpora la perspectiva de género de manera transversal en cada uno de los ejes que conforman el presente Plan” (Gobierno de la República, 2007, p. 211). Asimismo, se declara que "Construir políticas públicas con perspectiva de género de manera transversal en toda la Administración Pública Federal" (Gobierno de la República, 2007, p. 211) constituye una de sus tres estrategias transversales. En el PND (2013-2018) se sigue esta misma línea, y más aún, “incorporar la perspectiva de género” (Gobierno de la República, 2013, p. 10) se convierte en una de las tres estrategias transversales del gobierno federal de ese periodo.

En el ámbito educativo a nivel global, también la UNESCO ha tenido un rol fundamental al impulsar políticas públicas de educación con perspectiva de género en los distintos países (UNESCO, 2014a, 2014b). En México, la Secretaría de Educación Pública (SEP) y el Instituto Nacional de las Mujeres (InMujeres) firmaron en 2008 un convenio para promover la transversalidad de la equidad de género, así como la prevención, atención, sanción y erradicación de la violencia hacia las mujeres en el Sistema Educativo Nacional (OEI, 2008). Asimismo, se observa cierto interés de la SEP por dejar plasmada una perspectiva a favor de la equidad de género a lo largo del Programa Sectorial de Educación (PSE) del periodo 2013-2018. Inclusive en dicho periodo dentro de ese programa la estrategia transversal \#3 era la "Igualdad de Oportunidades y no Discriminación contra las Mujeres" (SEP, 2013, p. 47).5

En el ámbito educativo, el concepto de transversalidad tiene su origen en las/os teóricas/os de la educación, principalmente académicos/as franceses/as (Barbier, 1997). Según Cardaci (2005) el concepto “temas transversales” ha sido adoptado para referirse a aquellos que deberían impregnar toda la práctica educativa y, por supuesto, estar presentes en las diferentes áreas curriculares. En inglés se utiliza el término crosscurricular, pero su significado no es tan amplio ni tan profundo como el de transversalidad en castellano, pues cross-curricular se refiere al curriculum solamente.

El concepto de transversalización de la perspectiva de género en la IES adoptado en este estudio, se refiere a los procesos que tienen como fin que la perspectiva de género atraviese todos los dispositivos educativos, pero también los sistemas, estructuras y procedimientos de la propia IES. Comprende la legislación y normatividad universitaria, decisiones de las

${ }^{5}$ La implementación práctica de las políticas a favor de la equidad de género por parte de la SEP es un tema fuera de los alcances de este artículo. 
autoridades sobre presupuestos, paridad de género en cargos de toma de decisiones y cuerpos colegiados, equidad en el uso de espacios y canchas deportivas, combate al acoso sexual y a la violencia de género (considerando también aquella que ejercen mujeres contra mujeres), así como el uso de un lenguaje incluyente y no sexista. Esta perspectiva debe atravesar también programas y dispositivos educativos, el curriculum explícito (incluyendo la incorporación de autoras a la bibliografía), pero también los mensajes que se emiten en el curriculum oculto, las ilustraciones y contenidos de materiales educativos, el acceso y uso de tecnologías, etc. Implica paridad en los liderazgos de equipos de investigación, así como una vigilancia constante ante los sesgos de género tanto en planteamientos epistemológicos como en el trabajo investigativo. En los procesos de transversalización de la perspectiva de género se requiere que tanto las/os diversas/os agentes educativas/os, autoridades y el resto del personal de la IES desarrollen una conciencia de género crítica (Montes-de-Oca-O’Reilly y Yurén, 2010), misma que es resultado de experiencias formativas. Éstas por lo general incluyen la exposición a discursos académicos sobre género que propicien la reflexión e incluso, desestabilizaciones subjetivas.

La transversalización de la perspectiva de género en las IES es el fin último de las/os estudiosas/os del género en este nivel educativo. Implica que quienes hacen trabajo de género no permanezcan en los márgenes de la Institución, sino que el resto del personal docente y no docente conozca y comparta esta perspectiva. De acuerdo con Munévar y Villaseñor (2005) para esta transversalidad deben hacerse visibles las jerarquías culturales con base en el sexo, intentar desarticular los sobreentendidos de género, así como evitar responsabilizar a todos los hombres por el sometimiento de las mujeres.

Además de producir conocimiento sobre el género, las IES deben promover la equidad de género en su interior (Buquet, 2011). La autora asegura que las dificultades ampliamente documentadas a las que se enfrentan las mujeres en ese ámbito deben atenderse en forma directa por las mismas IES. Con base en la premisa de que las inequidades provocadas por el ordenamiento de género no son reconocibles como tales al contar con mecanismos que las naturalizan, Buquet (2011) considera necesario llevar a cabo lo que ella llama una "sensibilización" para que se genere una reflexión a nivel personal sobre las relaciones inequitativas entre los diferentes colectivos. Señala que estas actividades deben estar orientadas a autoridades, funcionarios, personal administrativo y población estudiantil; pero es particularmente relevante la sensibilización del personal académico para que por medio de su práctica docente puedan transmitir valores, actitudes y comportamientos favorables a la equidad de género.

\subsection{Incorporación de la perspectiva de género en la educación superior}

Si bien la transversalización de la perspectiva de género es el objetivo último de las/os académicas/os del género como colectivo, en la práctica se ha requerido de mucho esfuerzo por parte de las/os mismas/os para lograr avances y lo que ha venido ocurriendo es una incorporación paulatina. Estudios en diversos países concuerdan en que durante los procesos de incorporación de la perspectiva de género en instituciones de educación superior se han experimentado resistencias ${ }^{6}$ al interior de dichas organizaciones (Alonso

\footnotetext{
${ }^{6}$ Según Alonso y Lombardo (2016), había programas de Maestría interdisciplinarios sobre género, pero no fue sino hasta que se implementaron políticas del Espacio Europeo de Educación Superior, y de un gobierno considerado como socialista (2004-2011) que se introdujeron por primera vez estudios de género y política en los programas de formación universitaria (equivalente a licenciatura en México). Las autoras concluyen que para impulsar la perspectiva de género se requiere un
} 
y Lombardo, 2016; Asián et al., 2015; Messer-Davidow, 2002; Moghadam, 2001; Morley, 2007; Romero, 2008; Verge, Ferrer-Fons y González, 2017).

En Estados Unidos, Messer-Davidow (2002) señala como parte de una revisión histórica de los esfuerzos por impulsar la perspectiva de género en las instituciones de educación superior, que en los primeros años los principales problemas que se enfrentaron en ese país fueron, por una parte, las ortodoxias intelectuales de las disciplinas académicas y por otra, el deseo de mantener la departamentalización que estructuraba la toma de decisiones y la distribución de los recursos de las universidades. Así, los cursos sobre género fueron más fácilmente aceptados por la comunidad académica y el personal administrativo que los programas, pues estos atravesarían el ámbito curricular, lo cual "pondría en riesgo" la autoridad de los departamentos en sus propias áreas de conocimiento. Por otra parte, eran más sencillos los trámites burocráticos para la impartición de cursos dentro de las mismas unidades académicas que en las unidades especializadas de estudios de las mujeres (Messer-Davidow, 2002).

De esa forma, la inclusión del enfoque de género en el curriculum universitario de ese país se ha ido alcanzando mediante programas de estudio interdisciplinarios y la posterior creación de unidades académicas (Makovski y Paludi, 1993, Messer-Davidow, 2002). La proliferación de los estudios de género en años recientes se debió a la gran demanda que han tenido por parte de las estudiantes principalmente (Bystydzienski, 2001; MesserDavidow, 2002). Según la National Women's Studies Association (NWSA) en Estados Unidos en 2007 existían 652 programas sobre estudios de las mujeres y género a nivel licenciatura, maestría, y doctorado (NWSA, 2007).

En México, como en otros países, los estudios de género son resultado de décadas de esfuerzo de una gran mayoría de académicas y también de algunos académicos. Según Cardaci, Goldsmith y Parada (2002), a partir del activismo de las mujeres en los sesentas y setentas, se inició la impartición de cursos y se fueron creando centros y programas de investigación. Estas investigadoras apuntan que las iniciativas del movimiento amplio de mujeres generaron los primeros cursos sobre estudios de la mujer en México fueron impartidos a mediados de los setenta en la Escuela Nacional de Antropología e Historia y en la Facultad de Ciencias Políticas y Sociales de la UNAM y fueron proliferando en los ochentas y noventas. Autoras/es como Tarrés (1996), Cardaci, Goldsmith y Parada (2002) y Cervantes (2009) argumentan que algunos frutos del trabajo de las/os académicas/os del género fueron líneas, programas y centros de investigación. ${ }^{7}$ Cardaci, Goldsmith y

contexto político favorable, además de legislación, estructuras e instrumentos. Por su parte, Asián, Cabeza y Sosa (2015) afirman que la Universidad de Valencia en 2008 "logró que se aprobase su propuesta de integración de estudios de género en los títulos de grado y que se comenzase a impartir una asignatura sobre Relaciones de Género en el curso académico 2011-2012, siendo pionera entre las universidades españolas" (p. 40).

${ }^{7}$ Entre estos se encuentran el Centro Universitario de Estudios de Género de la Universidad de Colima, Centro de Estudios de Género de la Universidad de Guadalajara, Centro de Investigación y Estudios de la Mujer de la Universidad Michoacana de San Nicolás de Hidalgo, Centro de Estudios de Género de la Benemérita Universidad Autónoma de Puebla, Centro Universitario de Estudios de Género de la Universidad de Nuevo León, Centro de Estudios de la Mujer de la Escuela Nacional de Trabajo Social de la Universidad Nacional Autónoma de México, Proyecto Universitario de Género de la Universidad Iberoamericana Golfo-Centro, Mujer Rural de El Colegio de Postgraduados en Ciencias Agrícolas, Programa Interdisciplinario de Estudios de la Mujer de El Colegio de México, Programa Interdisciplinario de Estudios de Género de la Universidad de Guadalajara, Programa Universitario de Estudios de Género de la Universidad Autónoma de Chiapas, Programa Universitario de Estudios de Género de la Universidad Autónoma de México, Programa de Estudios de Género de la Universidad de Sinaloa, Programa de Estudios de la Condición de la Mujer y Relaciones de Género de la Universidad Autónoma de Yucatán, Programa de Estudios de Género de la Universidad de Sinaloa, Línea de Género y Salud del Programa Salud y Sociedad de El Colegio de Sonora, Línea de Investigación Mujer, Desarrollo y Salud Reproductiva de El Colegio de la Frontera Sur (Cardaci, Goldsmith y Parada, 2002). La Maestría en Estudios de la Mujer de la Universidad Autónoma Metropolitana Unidad Xochimilco está reconocida por el CONACYT como programa de alto nivel en su Padrón 
Parada (2002) señalan que aunque ha habido un crecimiento de la docencia en estudios de género, existía una relativa ausencia formal en los curriculum de licenciatura. González (2009) afirma que los investigadores adscritos a los estudios de género en educación en México todavía están lejos de constituir una comunidad epistémica, pues se carece de un conjunto de definiciones, problemas y dispositivos para la investigación.

En otro estudio de Cardaci (2005), se aplicó una encuesta a integrantes de centros y programas de estudios de género y de mujeres, así como a coordinadores/as de licenciatura, investigadoras/es y docentes en IES mexicanas. La autora encontró que ninguna de las licenciaturas consideradas incorporaba transversalmente, ni consideraba los temas de género como ejes de alguna fase de la formación. Señala que la situación que se halló con más frecuencia fue que la incorporación de esos temas a los cursos no formaba parte de un grupo de acciones convergentes con las de docentes de la misma disciplina ni de otros semestres o licenciaturas. Otro de sus hallazgos fue que las asignaturas relacionadas con el tema de género reflejaban, una historia de negociaciones entre diversas instancias para abrir una determinada "parcela" en el currículum (Cardaci, 2005), lo que coincide con lo encontrado en Estados Unidos por Messer-Davidow (2000).

En cuanto a la experiencia para incorporación de la perspectiva de género en las universidades públicas dentro de los estados de la República Mexicana (como es el caso de la IES objeto de este estudio), Bermúdez (2012) describe algunos factores en el proceso para la creación del Programa de Equidad de Género en la Universidad de Ciencias y Artes de Chiapas (UNICACH) y señala que con el Programa se genera un nuevo campo de relaciones de poder con los grupos dominantes de la institución. La autora manifiesta que se trata de una iniciativa de política pública "aceptable", en el sentido de lo políticamente correcto, pero que impacta lentamente en los espacios y procesos universitarios, pues exhibe y busca transformar las relaciones desiguales de género que los mismos actores institucionales reproducen.

En otro estudio sobre la incorporación de la perspectiva de género en la Universidad Autónoma del Estado de México, Vélez (s/f) menciona varios obstáculos. Manifiesta que uno de ellos es la cultura, debido a que tanto los mandatos sociales como los prejuicios y creencias fomentan la discriminación de las mujeres en la ciencia y la política, por lo que quienes buscan integrar la perspectiva de género en los procesos académicos y administrativos tienen a la estructura organizacional y a muchos/as dirigentes en su contra.

Según se observa en los párrafos anteriores, varias investigadoras coinciden, tanto en el ámbito internacional como nacional, en que existen resistencias al interior de las instituciones para la transversalización de la perspectiva de género. También en diversos estudios se observa que ha sido más difícil incidir a nivel de licenciatura (pregrado) que en los posgrados.

Nacional de Posgrados de Calidad. La UAM-Xochimilco también ofrece un área de especialización en el Doctorado en Ciencias Sociales. La Especialización en Género y Educación de la Universidad Pedagógica Nacional se ha retomado en otras unidades de la UPN en varios estados de la República (González, 2009). 


\section{Método}

Ante la imposibilidad de cubrir el enorme vacío sobre el tema, se optó por realizar una primera exploración examinando el caso específico de las/os directivos/as de una IES de México para conocer sus representaciones sobre la perspectiva de género, así como sus disposiciones para transversalizar, o en su caso, incorporar dicha perspectiva en su institución.

Para el procedimiento de selección de los/as colaboradores/as se observó que las unidades académicas (facultades y escuelas) de la IES están divididas en siete amplios campos del conocimiento. En cada campo se agrupan licenciaturas, posgrados, así como centros de investigación. Con el fin de que todos los campos de estudio de la institución estuvieran representados, se entrevistó a un hombre y a una mujer de cada uno de ellos. En total, se entrevistó a 14 directivos/as: siete mujeres y siete hombres, quienes son responsables de la reestructuración de planes y programas de estudio, así como eventos académicos, entre otras decisiones. Así pues, se seleccionó a quienes fueran o bien directores/as de facultad o centro de investigación, o bien secretarios/as académicos/as que tuvieran amplio conocimiento de la dinámica de la institución. Dos personas entrevistadas ocupan el cargo de la Secretaría Académica. La Secretaría Académica es la segunda posición con respecto a la Dirección de una Facultad dentro de esta IES. A excepción de una persona, toda/os son mexicanas/os.

El instrumento que se utilizó para la recolección de datos fue la entrevista semiestructurada con grabación de audio para su posterior transcripción. Una persona entrevistada no aceptó que se le grabara, por lo que a medida que hablaba, se iban escribiendo sus respuestas en papel. Todas las entrevistas se llevaron a cabo en las oficinas de los/as informantes, con excepción de una persona, que prefirió que la entrevista tuviera lugar fuera de su unidad académica. En el cuadro 1 se indica el número de hombres y mujeres entrevistadas/os que cuenta con algún título en los diversos niveles académicos.

Cuadro 1. Nivel educativo de las/os informantes

\begin{tabular}{lccc}
\hline & DOCTORADO & MAESTRÍA & LICENCIATURA \\
\hline Hombres & 2 & 2 & 3 \\
Mujeres & 5 & 1 & 1 \\
\hline
\end{tabular}

Fuente: Elaboración propia.

Del cuadro anterior se observan desde una perspectiva de género las diferencias en los niveles educativos de los/as colaboradores/as. Los hombres tienen en lo general, un nivel educativo menor que las mujeres. Cinco de las siete mujeres cuentan con doctorado y tres de los siete hombres tienen licenciatura.

Para los fines de este artículo, los/as colaboradores/as han sido identificados/as según su sexo, en donde M (Mujer) y H (Hombre). A cada uno/a se les ha asignado un número del 1 al 7 para diferenciarlos/as del resto de entrevistados/as de su mismo sexo. También se indica el grado de nivel de estudios alcanzado: D (Doctorado), M (Maestría) y L (Licenciatura) ${ }^{8}$. Así, H1M representa al hombre 1, mismo que cuenta con estudios de maestría y M6D indica la mujer 6, misma que cuenta con estudios de doctorado.

\footnotetext{
${ }^{8}$ En México, la licenciatura corresponde al nivel universitario e incluye a las ingenierías.
} 
El guion de entrevista incluyó preguntas como: ¿Qué es la perspectiva de género? ¿Existe equidad de género en la institución? ¿Considera que la perspectiva de género se encuentra incorporada en la institución? ¿De qué forma? ¿Existen obstáculos para la incorporación de la perspectiva de género? ¿Qué sugerencias tendría usted para transversalizar, o bien, incorporar la perspectiva de género en su unidad académica?

Para la interpretación de los resultados se utilizó el método de la teoría fundamentada (Glaser y Strauss; 1967) en combinación con la analítica de las representaciones (Jodelet, 1999). De la teoría fundamentada, se usó la variante del método comparativo constante. Esto es, se codifican y analizan datos simultáneamente para de esta forma desarrollar conceptos. Por medio de la constante comparación de determinados incidentes de los datos, se refinan dichos conceptos, se identifican sus propiedades, se exploran sus interrelaciones con el fin de integrar una teoría coherente (Taylor y Bogdan, 1987). Se iban comparando las declaraciones del/a colaborador/a con el discurso general que se maneja en los textos académicos sobre género. Las categorías fueron construidas a partir de lo que iban revelando los datos en términos de similitudes mayoritarias de respuesta.

\section{Resultados}

Las categorías que surgieron a partir de los datos analizados son las siguientes: representaciones sobre perspectiva de género, inequidades de género en el contexto social, representaciones sobre equidad de género en la institución, representaciones sobre inequidad de género en esa IES, contradicciones sobre si está incorporada o no la perspectiva de género en esa IES, resistencias para incorporar la perspectiva de género y sugerencias para la transversalización.

\subsection{Representaciones sobre perspectiva de género}

En términos generales, se observa un nivel bajo de conocimiento en los/as colaboradores/as sobre lo que es la perspectiva de género. Las mujeres muestran un mayor interés en este tema que los hombres, con excepción de uno de ellos. En varios casos, más que respuestas a las preguntas, lo que revelan las entrevistas son sus reflexiones sobre el tópico.

Las personas entrevistadas no tienen una visión histórica de cómo se han ido generando las políticas públicas sobre equidad de género y cuáles son los grupos que las han promovido. Se observa un abierto rechazo al feminismo en todos/as los/as participantes, excepto en una de ellas, que, aunque en sus clases da un espacio para la teoría crítica literaria feminista, considera que se trata de una filosofía "radical".

En este sentido, existe una tendencia a ofrecer un discurso políticamente correcto, de parecer "neutral", "en el medio, y apartado de los extremos" que es la representación que ellas/os tienen del feminismo. Aquí se presenta un fragmento de la respuesta de una de las mujeres participantes:

To creo que tenemos que tener mucho cuidado en esta perspectiva [de género], porque desde mi punto de vista hablar de género es hablar de hombre y mujer, generalmente se tiene la tendencia a hablar más de la mujer. En un programa de radio se hacía alusión a todas estas instituciones que se han creado alrededor de la mujer y que ponen a la mujer como... Siguen reforzando esta parte de sexo débil como si no fuera ella suficiente para seguir adelante, o para enfrentarse a ciertas situaciones. Con esto no quiero decir que efectivamente no ocurran ciertas situaciones en contra de la mujer. Sin embargo, también está la otra parte. El hombre pareciera que es el malo, pareciera 
que es siempre el agresor. $\Upsilon$ desde mi punto de vista ahora el desprotegido es el hombre. Para mí, hablar de género es hablar de una relación humana natural, reconociendo las diferencias de hombre y mujer, pero creo que ahorita se está inclinando la balanza hacia el otro extremo, del lado de la mujer. Entonces para mi es la integración, la integración de los dos sexos, ¿no? de los dos géneros. (M5D)

Algunos hombres tienen un discurso políticamente correcto a favor de la perspectiva de género. Sin embargo, durante la entrevista se observa que no conocen en qué consiste la perspectiva de género en realidad. Aquí un ejemplo:

\begin{abstract}
Bueno, yo entiendo por perspectiva de género como la igualdad o la definición de las diferentes actividades tanto de los hombres como de las mujeres, y aquí, retomando lo que hemos podido ver... ¿no? De repente es una situación que se tiene que dar, es necesaria pero siempre como que guardando los roles, ¿̇no? Porque hay un rol fundamental. El ser madre, ¿̇eh? Digo, no lo podemos hacer los hombres, ¿̇no? Aquí tenemos que atarlo con las situaciones sociales. La mujer tiene roles muy particulares pero muy importantes, ¿no? Si nos vamos a la base de la familia pues es la formadora propiamente tanto de los hijos como del mismo marido. Yo sí creo que atrás de un gran hombre hay una gran mujer, ¿̇no? (H5L)
\end{abstract}

Este participante piensa que deben guardarse los roles de género, que debe haber una diferenciación de las actividades de mujeres y hombres, que las mujeres deben formar a los hijos y a su marido y que deben estar detrás de los hombres apoyándolos. Todo ello es contrario al discurso de equidad de género que manejan la/os teórica/os.

La mayoría de la/os colaboradora/es hace declaraciones contradictorias. En vario/as, su discurso es políticamente correcto, es decir, se manifiestan a favor de la equidad de género. Sin embargo, el análisis saca a relucir discursos y prácticas totalmente opuestos a ésta. Se advierte que la experiencia de género es un factor que incide directamente en la conciencia de género en estas/os académicos/as de educación superior. En este sentido, se observa que las mujeres tienen una mayor sensibilidad con respecto a las problemáticas de género que los varones. El único hombre que muestra conocimiento sobre género trabaja una disciplina que está relacionada con el tema. En general, la mayoría de quienes pertenecen a una disciplina en ciencias llamadas “duras” están menos sensibilizados/as hacia la perspectiva de género, que aquellas/os cuyas disciplinas tienen una mayor relación con este tópico.

Sólo dos colaboradores/as (un hombre y una mujer) -pertenecientes a disciplinas académicas que propician la reflexión sobre temas personales y sociales- tienen un discurso similar al de las/os teóricas/os del género. Ambos/as pudieron brindar un diagnóstico más preciso del estado de esta institución de educación superior en el tema de género.

\title{
3.2. Representaciones sobre inequidad de género en el contexto social
}

La mayoría de los/as entrevistados/as relaciona el concepto de género con los conceptos de igualdad y de equidad y enfatiza que el género no es sólo un asunto de mujeres, sino una cuestión de hombres y mujeres. Varios/as hacen referencia a inequidades del contexto social en que laboran, si bien eso no formaba parte de las preguntas del cuestionario. Dos hombres cuyas disciplinas académicas tienen relación con el campo señalan que, en comparación con la vida urbana, es en las zonas rurales donde tienen lugar las mayores inequidades. En sus palabras:

Nuestra formación tiene que ver con el sector rural y las peores injusticias de la equidad de género se dan ahí en ese sector y te lo digo con conocimiento de causa, con mucha tristeza porque ves coartadas muchas veces el desempeño y desarrollo de las 
mujeres, ¿̇no? Desde porqué no las dejan estudiar, porqué se casan muy jóvenes, porqué tienen que mantener ellas tanto la formación de la familia como el proveer los mismos alimentos. O sea, el trabajo en comunidad es muy duro para la mujer. (H5L)

En cuanto al estudiantado, vario/as participantes mencionaron haber visto violencia de género hacia las estudiantes. Un ejemplo a continuación de un participante que está familiarizado con temas de género: "Somos conscientes de que podemos estar engañados nosotros mismos, de que puede haber problemas subyacentes y no los detectamos. Los chicos sí hablan, los estudiantes. Hay problemas de agresividad del novio con la novia”. (H2D)

Otra entrevistada coincide:

Aqui en [menciona el estado de la República Mexicana] el machismo está muy, muy elevado. Es algo muy notorio. Lo vemos en las alumnas, como la violencia. Se casan muy chiquitas o tienen pareja muy chiquita y es lo que diga la pareja. (M4L)

\title{
3.3. Representaciones de equidad de género en la institución
}

La mayoría de los hombres entrevistados tiene una representación de equidad en esa IES. Un razonamiento común es que no hay ninguna regla que impida el desarrollo de las mujeres. Al no haber ningún lineamiento explícito en contra, su reflexión es que entonces existen las mismas oportunidades para todos/as. Aquí la declaración de un hombre:

\footnotetext{
Aquí en la [institución] yo no he visto inequidad. Aquí lo que te cuenta es tu preparación. Seas maestro o maestra tienes las mismas oportunidades. Si tú ganas en cuanto a tu puntaje, a tu capacidad, se te da la materia. (H5L)
}

Sin embargo, la mayor parte de las personas entrevistadas interpretó la pregunta de si había equidad en la institución con la equidad en el acceso a cargos de toma de decisiones. A continuación, las palabras de dos de los hombres participantes:

\begin{abstract}
En cuanto a puestos administrativos, pues sí, también hay un buen número. No sé cuál será el número, pero sí hemos visto que hay mujeres, ¿no? Entonces ahi es libre. Es un trabajo colegiado. Pero las voces se escuchan por igual. No hay nada, nada, ¿̨eh? Ni una pizca de discriminación. (H4M)

Dentro de la [institución] la cantidad de directivos mujeres y hombres... No tengo las estadísticas, pero uno percibe que hay muchas mujeres directivas, que se tienen en cuenta sus capacidades a la hora de nombrarlas para un cargo, o en otro. Eso yo creo que es muy importante, porque no en todas las instituciones se tiene esta proporción de mujeres dirigentes como hay en la [institución]. (H2D)
\end{abstract}

\subsection{Representaciones de inequidad de género en la institución}

Al contrario de lo observado en párrafos anteriores en palabras de los hombres, las expresiones más frecuentes de inequidad de género en esa IES fueron proporcionadas por las mujeres. El único varón familiarizado con el tema y cinco directivas mencionan que las posiciones más importantes en la institución son ocupadas por hombres. Una mujer manifiesta: "En la universidad yo encuentro una estructura muy vertical, entonces la mujer sí tiene espacios, pero es más los puestos de toma de decisiones, más por hombres" (M5D). A continuación, se presenta un fragmento de lo que piensa otra mujer:

Bueno, yo creo que es sintomático que no haya ni una sola mujer allá arriba, ¿̇no? en las esferas más altas. Ninguna secretaría la ocupa una mujer. Todos son hombres. De lo que recuerdo siempre han sido hombres. [Menciona tres secretarías]. La Secretaría General ni se diga, ésa siempre ha sido masculina. Todos. $\Upsilon$ la Rectoría desde luego. Nunca ha habido Rectora. Del Rector y los tres secretarios que son los cargos más importantes aquí en la [institución]. (M1D) 
La opinión del hombre mencionado anteriormente concuerda con la de la mayoría de las mujeres:

\begin{abstract}
Ya no podemos relacionarnos desde una postura autoritaria que tenemos los hombres y hay ejemplos dramáticos. Si usted me dice: “¿cuántas directoras ha habido de la facultad?” Ninguna. “¿Cuántas profesoras hay?” La mayoría. “¿Cuántas estudiantes?” La gran mayoría son mujeres. Pero aun así siguen siendo los hombres los que dirigen. Debemos dejar los hombres esta postura machista. (H1M)
\end{abstract}

La única mujer que se manifestó absolutamente en contra de la perspectiva de género reconoce ella misma que las posiciones más altas las ocupan los hombres aún en el campo de las ciencias “duras”. Sin embargo, le da a ello una explicación biológica, lo cual es contrario a las explicaciones que brindan las/os teóricas/os del género como construcción sociocultural:

Yo no siento diferencia. A lo mejor es el área en que nos movemos. $\Upsilon$ sí, es verdad que no llegamos a los altos puestos. Sí, es verdad que, a nivel de cúpulas, por ejemplo, cuándo ves... aún en la Ciencia. O sea, en la Ciencia sí se da. O sea, ¿̇quiénes son los jefes de laboratorio? Hay más jefes de laboratorio que jefas de laboratorio. Pero eso yo no creo que es por discriminación de los hombres hacia las mujeres. Ésa es una auto-discriminación. $O$ sea, en las mujeres también tenemos diferencias. Tampoco tenemos que olvidar las diferencias. Nuestra mayor prioridad en la vida biológica, o sea a nivel biológico es tener hijos. Eso lo tenemos bien metido en el fondo de nuestros genes. Entonces eso sí nos cambia las prioridades. $\Upsilon$ yo creo que al $80 \%$ de las mujeres les vale madre si llegas a ser directora o si llegas a ser lo que sea o grande funcionaria, o presidenta, porque lo que quieres en el fondo es tener hijos. Yo fui directora, pero no fue nunca mi prioridad en mi vida. Llegué por azares del destino, pero yo no luché por ser directora. To me sentí realizada cuando tuve a mis hijos y ahi la diferencia de los hombres. Los hombres no se sienten realizados teniendo un hijo. Pero el $80 \%$ de los hombres... ellos quieren destacar. Está dentro de sus genes. Los hombres evolutivamente han estado seleccionados para sobresalir. Lo ves en la naturaleza. Los machos alfa son los que sobresalen. ¿Cómo sobresalen? Luchando entre ellos, siendo el jefe de la manada. O sea, están evolutivamente programados para sobresalir, ¿̇por qué? Porque sobresalir es la forma de asegurarse de que se van a poder aparear y dejar descendencia. Así se ha seleccionado pa' que los hombres así se comporten. Entonces ¿̇qué quieren los hombres? Llegar a ser directores, presidentes, manejar, manejar a todos. Eso quieren los hombres. Intelectualmente los dos somos iguales, los dos somos capaces de llegar a altos puestos, de ser doctores o lo que sea. Pero una mujer a la hora de pelearse por un puesto, una mujer va a decir: “iNo! ¿ Yo? ¡Qué hueva! To prefiero irme temprano, ir por mis hijos que pasarme todo el día peleándome en reuniones inútiles" (que además te puedo decir que son inútiles), peleándose por idioteces. "Prefiero ganar menos, pero estar tranquila, no tener tantas presiones y tener tiempo para mi familia”. (M6D)

En el caso de otra participante, percibe equidad en su facultad, pero inequidad en los altos cargos de la institución.

Me parece que hay igualdad. Posiblemente no en los cargos administrativos, a nivel de la administración central ahí prácticamente la mayoría son hombres, pero ya en la comunidad académica, me parece que no he percibido diferencias. El trato es similar.

$(\mathrm{M} 2 \mathrm{D})$

Todas las mujeres proporcionaron ejemplos de inequidad en la institución. Sin embargo, sus representaciones de esa inequidad y las razones de ésta varían considerablemente de mujer a mujer. Algunas mencionaron varias situaciones de inequidad que han vivido en el ámbito profesional o en el privado. En seguida, una mujer expresa que ha vivido rechazo como mujer directiva por parte de integrantes de su propia unidad académica: 
To lo vivo en mi facultad. Esta facultad habia sido siempre dirigida por hombres. Muchos maestros aceptan el cambio, me reconocen. Pero noto mucha resistencia en algunos otros [dicen]: "nombre, ahora todos estamos bajo el orden del matriarcado". Trato de ser equitativa. La discriminación que muchas veces se siente. (M3L)

De esta manera, el fenómeno que las teóricas llaman "el techo de cristal" es advertido por las colaboradoras y por un colaborador que por su trabajo tiene relación con el tema de género, si bien ninguna/o menciona ese concepto. Cabe destacar que la mayoría de los hombres percibe que existe equidad de género en la institución, mientras que la mayor parte de las mujeres piensa lo contrario, que no existe equidad en la misma.

\title{
3.5. Representaciones contradictorias sobre la incorporación de la perspectiva de género en la institución
}

A la pregunta sobre si está incorporada la perspectiva de género en la institución, la mayoría de mujeres (seis) y un varón respondieron que no está incorporada, o bien que se encuentra incorporada en forma muy incipiente. Tres varones dijeron que sí está incorporada. Otros tres hombres hicieron declaraciones contradictorias. Uno de ellos dijo que sí estaba incorporada la perspectiva de género en la institución y después comentó que no era así. El otro participante al principio expresó que no y posteriormente declaró que sí. La mujer restante no supo contestar porque manifestó no entender qué significa perspectiva de género.

En general, existe la representación de que ciertas políticas públicas para incorporar la perspectiva de género provienen del gobierno federal. Algunos/as participantes consideran esto más bien como una "imposición" sin que la comunidad universitaria entienda bien cómo y por qué hacerlo. Un participante lo expresa de esta manera:

\begin{abstract}
Es cierto que en el Plan Institucional ${ }^{\circ}$ está por ahí señalado que la [institución] deberá atender a la diversidad, pero todavía no hay nada concreto. Es cierto también que desde lo institucional se está impulsando. Este año tuvimos la oportunidad de desarrollar foros de equidad de género en todas las áreas, pero más bien son como cuestiones de orden institucional que hay que hacerlo, ¿no? Es cierto también que hoy estamos integrando al [nombra un programa de financiamiento gubernamental a las universidades] por una cuestión de género, pero también es más como seguir los lineamientos. (H3L)
\end{abstract}

Varios/as entrevistados/as señalaron que, por requerimientos de la planeación financiera de la institución, tuvieron que incorporar la perspectiva de género en el presupuesto para actividades académicas de ese año. Cabe señalar que una directiva que labora en el campo de las ciencias "duras" no supo cómo hacerlo:

\begin{abstract}
No entiendo eso. O sea, cuando hablan de que hay que integrar la perspectiva de género a los programas educativos no entiendo qué quieren decir. No me queda claro. Ahora que tuvimos que hacer el [nombra un programa de financiamiento gubernamental a las universidades]. El chiste es que es una bolsa donde las universidades participan para tener fondos para apoyar los programas educativos. Se juntan las diferentes facultades (o como se llamen), hacen su programa de desarrollo y entonces piden dinero. Te ponen puntitos. Si se está incorporando la perspectiva de género, si se ve el género en no sé qué. $\Upsilon$ yo tuve que hacer el [nombra un programa de financiamiento gubernamental]. La verdad no me queda claro a qué se refiere incorporar perspectiva de género en programas educativos. O sea, no entiendo. No entiendo qué quiere decir. (M6D)
\end{abstract}

${ }_{9}$ Por razones de confidencialidad se ha cambiado el nombre del Plan. 
Los párrafos anteriores revelan una aplicación inadecuada de una política pública correcta. Se emite la orden de incorporar la perspectiva de género en el presupuesto de la IES desde el Gobierno Federal, pero no se proporciona la capacitación ni sensibilización pertinentes. El resultado es que los/as directivos/as cumplen con el requisito, pero el recurso económico no se utiliza en forma eficiente y los resultados son pobres.

\subsection{Resistencia a la incorporación de la perspectiva de género}

Cuatro hombres y dos mujeres entrevistadas mencionaron resistencia hacia dentro de la institución para incorporar la perspectiva de género por parte de autoridades, órganos colegiados, profesores/as, personal administrativo, etc. Aquí un hombre manifiesta que no existe interés real dentro de la institución para impulsar el enfoque de género:

En pláticas que hemos tenido con directores, o con cuerpos académicos, o con investigadores realmente no hay una creencia en la perspectiva de género. Creo que la habilidad del macho es como reflexionar mucho para no hacer nada. Es como programar una sesión, luego uno la boicotea, entonces la pasamos para la siguiente semana. $\Upsilon$ después para la siguiente semana ya están otros proyectos más importantes. $\Upsilon$ se va posponiendo, y se va posponiendo. Administrativamente es fácil utilizar las cosas para posponerlas, para boicotearlas, para reventarlas. $(\mathrm{H} 1 \mathrm{D})$

A continuación, se muestra lo que opina otro entrevistado, que manifiesta que se sigue un discurso políticamente correcto en torno al género, pero que cuando debe pasarse a las acciones, estas son boicoteadas:

Los profesores también somos eminentemente machistas. Habrá que dialogarlo mucho con los profesores. A veces discursivamente lo decimos y esto es muy común. En toda la [institución] yo escucho los discursos sobre el género, pero cuando se trata de hacer algo en concreto es cuando decimos, "no pues pérame [sic], no tengo tiempo, no puedo. Digo, políticamente tiene que hacerse, ¿no? y nos pasa a todos. Nos pasa en muchas ocasiones. (H3L)

Lo que las declaraciones de estos tres hombres revelan es que, aunque la política pública indique que debe incorporarse la perspectiva de género, hacia adentro de la institución existen resistencias por parte de los/as propios directivos/as, investigadores/as y profesores/as. Lo mismo sucede con la creación de cursos o seminarios sobre género. El participante que está más familiarizado con el tema expone lo siguiente:

Si yo la quiero meter como una materia, es rechazada por la gran mayoría de los catedráticos [ello preguntaría:] “¿por qué género y por qué no violencia sexual? ¿̇ por qué no comunidad y país? ¿̇o por qué no...?” Cada capillita ${ }^{10} .$. Tendríamos entonces una lista de materias que un estudiante no terminaría nunca porque en toda institución hay cotos de poder. En todas las instituciones hay las famosas tribus. Si uno mete una propuesta, las otras tribus dicen "¿y por qué yo no?, entonces [tendríamos] el chorizo ${ }^{11}$ de materias y la tendencia ahora es que sea más corta, ¿̇no? (H1M)

Así, para este participante, un obstáculo para la creación de nuevos cursos sobre género son los cotos de poder dentro de la propia comunidad académica. En este sentido, cuando se refiere a cada "capillita", su visión coincide con lo encontrado por autoras como Cardaci (2005) que denomina "parcelas" a dichos cotos.

\footnotetext{
${ }^{10}$ Se refiere a cada coto de poder.

${ }^{11}$ Hace referencia a un embutido de carne de cerdo de considerable longitud, que se consume en España, México y otras regiones de América.
} 
Otro ejemplo que revela las resistencias que existen hacia dentro de la institución es el proceso para la creación de un Protocolo de Atención a la Violencia de Género. El mismo participante comenta que si bien el protocolo está disponible, las víctimas de violencia no se atreven a denunciar debido a que el protocolo fue elaborado por los abogados de la institución, quienes no consultaron a las investigadoras especialistas en género, por lo que el protocolo tiene ciertas fallas graves, como la revictimización de quienes vivieron violencia. Desde su punto de vista, las víctimas no denuncian, o bien, denuncian muy poco debido a que el protocolo indica que deben volver a estar en presencia de la supuesta parte agresora durante el proceso.

\subsection{Sugerencias para transversalizar la perspectiva de género}

Para la transversalización de la perspectiva de género en la institución, algunas/os colaboradoras/es opinan que debe partirse "de arriba hacia abajo". Es decir, que se debe empezar por el convencimiento de las autoridades para que éste permee al resto de la estructura institucional. Por el contrario, otros/as opinan que se debe partir "de abajo hacia arriba”. Es decir, afirman que es desde el estudiantado, profesorado y personal administrativo de donde debe partir para llegar a convencer a los estratos superiores de la institución. Aquí un ejemplo: "Entonces yo creo que hay que construir la entrada de la perspectiva de género. Que no surja por generación espontánea... Empezar con un programa de género en cada unidad académica" (H1M), Otro entrevistado comenta:

Como que a mí me parece que este asunto tendría que despegar desde la base, ¿no? Desde la gente que está ahí. Tendría que empezar a trabajar eso, porque desde lo institucional pues funciona, pero funciona en el momento y desaparece. $(\mathrm{H} 3 \mathrm{~L})$

$\mathrm{Al}$ preguntar qué sugerencias tenían para la transversalidad de la perspectiva de género, la mayoría de las/os entrevistadas/os sugirieron talleres. Las/os dos colaboradores/as que tienen más experiencia en el trabajo en este tema coincidieron en que los talleres son el medio más adecuado para incorporar la perspectiva de género a esta IES debido a que los talleres -más que las conferencias o ponencias- propician una reflexión de las propias experiencias de vida. Ella señala la importancia de los talleres, que están mucho más cerca de lo vivencial:

No basta con hacer aquí grandes congresos, encuentros porque no están dadas las condiciones para que eso logre permear. Creo que hay que ir más abajo, más a la base, en las clases, aquí con los estudiantes, en una optativa, hacerlo transversal. No basta un encuentro esporádico allá una vez cada semestre. Es un tema fundamental. (M1D)

El varón que ha trabajado durante varios años esta problemática coincide en la pertinencia de los talleres, pero añade que es importante que éstos tengan valor curricular:

Yo confío mucho en los talleres. No creo en las conferencias. Después de la conferencia que dura dos horas, la gente se va y no... La perspectiva de género... yo creo que tiene que ser por talleres dentro de las unidades académicas, donde se reflexione de manera seria, pero hacia el interior, no tanto de si hemos recibido violencia, sino cómo ejercemos nosotros la violencia. Creo que sería uno de los primeros puntos. A ver: ¿cómo ejerzo la violencia desde este cubículo? Está la secretaria académica, no respeto su tiempo. Utilizo a la otra compañera... ¿Cómo yo en lo concretito, desde que llego? Sí. Me doy cuenta. ¿Cómo controlo? ¿Cómo manipulo? ¿Cómo seduzco para que las cosas giren desde mi punto de vista? En el fondo de todo está el ejercicio del poder. En todo, en lo que me mencione. Siempre hay un ejercicio del poder. $Y$ como las condiciones están dadas para que el hombre las pueda ejecutar, pues se aprovecha. El ejercicio del poder cuando se abre, agarra otras dimensiones. Hay ejercicio del poder simulado -que es lo que se utiliza mucho: la simulación-y hay ejercicio del poder radical, que también se da, ¿̇no? (M1M) 
Otras sugerencias por parte de amba/os es la creación de órganos de difusión dedicados a este tema y el uso de material audiovisual "pequeños videos de YouTube, cosas vivenciales. Si no, la gente no se apropia” (M1D). Sobre la incorporación de esta perspectiva al curriculum, estas dos personas, así como otra mujer, se pronunciaron por introducirla como curso optativo. El resto de la/sos participantes no mencionó propuestas concretas. Ninguna/o habló de transversalización.

\section{Conclusiones}

En términos generales se advierte poco conocimiento en los/as entrevistados/as sobre lo que es la perspectiva de género. Un razonamiento común, particularmente en los hombres, es que no hay ninguna regla que impida el desarrollo de las mujeres. Al no haber ningún lineamiento explícito en contra, la lógica de varios/as colaboradores/as es que entonces existen las mismas oportunidades para todos/as.

En varios/as, su discurso es políticamente correcto, es decir, se manifiestan a favor de la equidad de género. Sin embargo, el análisis revela discursos y prácticas opuestos a ésta. Existe una tendencia a ofrecer un discurso políticamente correcto en la neutralidad, en el justo medio, apartado de lo radical y de los extremos. Se observa un abierto rechazo al feminismo en todos/as los/as participantes, con excepción de una, quien, no obstante, percibe a éste como radical.

Se advierte que el sexo de las personas entrevistadas es un factor que incide directamente en la conciencia de género de estas/os directivas/os. En general, las mujeres tienen mayor interés y una mayor sensibilidad con respecto a las problemáticas de género que los varones. La mayoría de ellas percibe inequidad de género en esta institución, al contrario que la mayoría de los hombres. Todas ellas proporcionan ejemplos de inequidad en la institución y algunas mencionan experiencias propias. En ese tenor, el fenómeno que las teóricas llaman el techo de cristal, aunque no con ese nombre, es advertido por las colaboradoras y por un colaborador que por su trabajo tiene relación con el tema de género.

Sólo dos personas entrevistadas (un hombre y una mujer) -pertenecientes a disciplinas académicas que propician la reflexión sobre temas personales y sociales- tienen un discurso similar al de las/os teóricas/os del género. Ambo/as pudieron brindar un diagnóstico más preciso de la situación de esta institución de educación superior en el tema de género, así como sugerencias para la implementación de un programa de esta naturaleza, como es la impartición de talleres vivenciales que resulten en una reflexión de las/os participantes.

Si bien la política pública indica que debe incorporarse la perspectiva de género, en la práctica hacia adentro de la institución existen resistencias por parte de autoridades, investigadores/as, profesores/as y personal administrativo. Lo mismo sucede con la creación de cursos o seminarios sobre género. Un obstáculo para la creación de nuevos cursos sobre género son los cotos de poder dentro de la propia comunidad académica.

Los/as dos colaboradores/as que tienen más experiencia en el trabajo en este tema coincidieron en que los talleres son el medio más adecuado para incorporar la perspectiva de género a esta institución de educación superior debido a que los talleres -más que las conferencias o ponencias- propician una reflexión de las propias experiencias de vida. 
Amba/os son la creación de órganos de difusión dedicados a este tema y el uso de material audiovisual. Sobre la incorporación de esta perspectiva al curriculum, estas dos personas, así como otra mujer, se pronunciaron por introducirla como curso optativo. El resto de las/os colaboradoras/es no mencionó propuestas concretas y en lo general se observó escaso interés para ello.

Además de las sugerencias para la transversalización de la perspectiva de género que se han discutido en la literatura (y que también aparecen en el apartado sobre transversalización de este artículo) y dado que muchos planteamientos a favor de la equidad de género provienen de los diversos feminismos académicos, sugiero que en los planes de transversalización de la perspectiva de género se considere de manera prioritaria una comunicación efectiva que aclare qué sí son y qué no son los feminismos. Ello conllevaría también diferenciar los feminismos académicos de los feminismos surgidos de la esfera activista. Pudiera ser que algunas manifestaciones que surgen de esta esfera sean luego difundidas en forma negativa, lo que resulta en representaciones de los feminismos ajenas a lo que éstos postulan. Asimismo, resultaría pertinente que se explicara desde una visión histórica que las políticas públicas a favor de la equidad de género, no ocurren por iniciativa de los gobiernos en sí, sino después de muchos esfuerzos de grupos organizados, mayoritariamente compuestos por mujeres.

\section{Referencias}

Acker, S. (1994). Gendered education: Sociological reflections on women, teaching, and feminism. Buckingham: Open University Press.

Alonso, A. y Lombardo, E. (2016). Ending ghettoization? Mainstreaming gender in Spanish political science education. European Political Science, 15(3), 292-302. https://doi.org/10.1057/eps.2015.77

Anderson, E. (1995). Feminist epistemology: An interpretation and a defense. Hypatia, 10(3), 5084. https://doi.org/10.1111/j.1527-2001.1995.tbo0737.x

Anderson, E. (2017). Feminist epistemology and philosophy of science. The Stanford encyclopedia of philosophy. Recuperado de https://plato.stanford.edu

Asián, R., Cabeza, F. y Sosa, V. (2015). Formación en género en la universidad. ¿Materia de asignaturas específicas o de educación transversal? Revista Historia de la Educación Latinoamericana, $17(24)$, 35-54. https://doi.org/10.19053/01227238.3310

Barbier, R. (1997). L'approche transversale: L'écoute sensible en sciences humaines. París: Édition Anthropos.

Bermúdez. F. (2012). Transversalidad de género en el ámbito universitario. El caso de la Universidad de Ciencias y Artes de Chiapas (UNICACH). Géneros. Revista de investigación y Divulgación sobre los Estudios de Género, 19(11), 37-55.

Bourdieu, P. (2000). La dominación masculina. Barcelona: Anagrama.

Buquet, A., Cooper, J., Rodríguez, H. y Botello, L. (2006). Presencia de mujeres y hombres en la UNAM: Una radiografía. Ciudad de México: UNAM-PUEG.

Buquet, A. (2011). Transversalización de la perspectiva de género en la educación superior. Problemas conceptuales y prácticos. Perfiles Educativos, 33, 211-225. 
Bustos, O. (2008). Los retos de la equidad de género en la educación superior en México y la inserción de mujeres en el mercado laboral. ARBOR Ciencia, Pensamiento y Cultura, 18(3), 795-815.

Bystydzienski, J. (2001). Programas de estudios de la mujer en los Estados Unidos: Historias y temas actuales. En E. Zapata, V. Vázquez y P. Alberti (Coords.), Género, feminismo y educación superior: una visión internacional (pp. 67-99). Ciudad de México: MIAC.

Cardaci, D., Goldsmith, M. y Parada, L. (2002). Los programas y centros de estudios de la mujer y de género en México. En G. Gutiérrez (Ed.), Feminismo en México: Revisión histórico-crítica del siglo que termina (pp. 98-109). Ciudad de México: PUEG-UNAM. https://doi.org/10.2307/j.ctv47w9dv.20

Cardaci, D. (2005). ¿Ausentes o invisibles? Contenidos sobre las mujeres y los géneros en el currículo de licenciatura de universidades mexicanas. La Ventana. Revista de Estudios de Género, 6(1), 107-142. https://doi.org/10.22201/crim.unamo00001c.2017.c32

Castro, R. y Vázquez, V. (2008). La universidad como espacio de reproducción de la violencia de género. Un estudio de caso en la Universidad Autónoma de Chapingo, México. Estudios Sociológicos, 26(78), 587-616. https://doi.org/10.20983/noesis.2013.3.1

Cervantes, C. (2009). Los estudios de género y las redes académicas de coordinación interinstitucional en México. En E. Molina Bayón y N. San Miguel (Coords.), Estudios de género y desarrollo: Balance y propuestas (pp. 35-67). Madrid: Universidad Autónoma de Madrid. https://doi.org/10.2307/j.ctv47w9dv.20

CONACyT. (2019). Padrón de beneficiarios del SNI. Recuperado de www.conacyt.gob.mx/images/SNI/Vigentes_Enero_2019.xlsx.

García, P. (2004). Mujeres académicas: El caso de una universidad estatal mexicana. Ciudad de México: Plaza y Valdés.

García, P. (2005). Género, educación y política pública. La Ventana. Revista de Estudios de Género, $21(2), 70-89$.

Glaser, B. y Strauss, A. (1967). The discovery of grounded theory: Strategies for qualitative research. Chicago, IL: Aldine. https://doi.org/10.1097/00006199-196807000-00014

Gobierno de la República. (2001). Plan nacional de desarrollo (2001-2006). Recuperado de http://www.sagarpa.gob.mx/ganaderia/Publicaciones/Lists/Otros/Attachments/4/PN Do106.pdf

Gobierno de la República. (2007). Plan nacional de desarrollo (2007-2012). Recuperado de http://pnd.calderon.presidencia.gob.mx/pdf/PND_2007-2012.pdf

Gobierno de la República (2013). Plan nacional de desarrollo (2013-2018). Recuperado de http://pnd.gob.mx/

González, R. (2009). Estudios de género en educación: Una rápida mirada. Revista Mexicana de Investigación Educativa, 14(42), 681-699.

Harding. S. y Norberg, K. (2005). New feminist approaches to social science methodologies: An introduction. Signs: Journal of Women in Culture and Society, 30(4), 2009-2015. https://doi.org/10.1086/428420

Jodelet, D. (1999). La representación social: Fenómenos, concepto y teoría. En S. Moscovici (Coord.), Psicología social II. Pensamiento y vida social (pp. 469-494). Barcelona: Paidós.

Lamas, M. (2000). Diferencias de sexo, género y diferencia sexual. Cuicuilco. Nueva Época, 7(18), 124. 
Longino, H. (1993). Subjects, power and knowledge: Description and prescription in feminist philosophies of science. En L. Alcoff(Ed.), Feminist epistemologies (pp. 101-120). Nueva York, NY: Routledge.

Makovski, V. y Paludi, M. (1993). Feminism and women's studies in the academy. En M. Paludi y G. Steuernagel (Eds.), Foundations for a feminist restructuring of the academic disciplines (pp. 137). Londres: The Haworth Press. https://doi.org/10.4324/9780203825105

Messer-Davidow, E. (2002). Discipliningfeminism: From social activism to academic discourse. Durham, NC: Duke University Press. https://doi.org/10.1215/9780822383581

Moghadam, V. (2001). Institutionalizing and globalizing women's studies at Illinois State University. Gender Issues, 19(2), 5-15. https://doi.org/10.1007/s 12 147-001-00 14-9

Montes-de-Oca-O’Reilly, A. y Yurén, T. (2010). Trayectancia y formación de liderazgo en académicas mexicanas: El caso Morelos. Archivos Analíticos de Políticas Educativas, 18(12), 131. https://doi.org/10.14507/epaa.v18n12.2010

Morley, L. (1994). Glass ceiling or iron cage: Women in UK academia. Gender, Work and Organization, 1(4), 194-204. https://doi.org/10.1111/j.1468-0432.1994.tboo018.x

Morley, L. (2007). Sister-matic: Gender mainstreaming in higher education. Teaching in Higher Education, 12(5), 607-620. https://doi.org/10.1080/13562510701595267

Munévar, D. y Villaseñor, M. (2005). Transversalidad de género. Una estrategia para el uso político-educativo de sus saberes. La Ventana. Revista de Estudios de Género, 3(21), 44-68. https://doi.org/10.24201/eg.v2i4.50

NWSA. (2007). Mapping women's and gender studies data collection executive summary. Recuperado de http://www.nwsa.org/files/CensusExec_Summary-2.pdf

OEI. (2008). Acuerdo SEP e Inmujeres para promover la perspectiva de género en educación. Recuperado de http://www.oei.es/noticias/spip.php?article1929.

ONU. (1995). Report of the fourth world conference on women. Recuperado de: http://beijing20.unwomen.org/ / media/Field\%20Office\%20Beijing\%20Plus/Attachmen ts/BeijingDeclarationAndPlatformForAction-en.pdf

Otto, D. (2010). Power and danger: Feminist engagement with international law through the UN security council. Australian Feminist Law Journal, 32(1), 97-121.

https://doi.org/10.1080/13200968.2010.10854439

PNUD Colombia. (2005). Estrategia equidad de género. Recuperado de http://www.pnud.org.co/img_upload/196a010e5069fodbo2ea92181c5b8aec/Estrategia\% 2ode\%20genero\%20PNUD\%2oColombia.pdf

PUEG. (2010). Diagnóstico de la situación de mujeres y hombres por dependencia. Ciudad de México: Instituto de Matemáticas de la UNAM. https://doi.org/10.2307/j.ctt2204qk3.5

Rodríguez, C. (2016). El sistema nacional de investigadores en números. Recuperado de http://www.foroconsultivo.org.mx/libros_editados/SNI_en_numeros.pdf

Romero, L. (2008). La transversalización de género en la educación: ¿Qué pasa en las escuelas de Galvarino, la capital indígena de la región de La Araucanía? Segunda Época. Revista de Estudios de la Mujer, 12, 21-32. https://doi.org/10.2307/j.ctv47w4no.15

Rose, H. (1983). Hand, brain, and heart: A feminist epistemology for the natural sciences. Signs: Journal of Women in Culture and Society, 9(1), 73-90. https://doi.org/10.1086/494025 
Rosser, S. (1992). Are there feminist methodologies appropriate for the natural sciences and do they make a difference? Women's Studies International Forum, 15(5), 535-550.

https://doi.org/10.1016/0277-5395(92)90057-3

Sánchez M. (octubre, 2008). ¿Qué tanto es tantito? El hostigamiento sexual. El caso de las trabajadoras administrativas de la UNAM. Ponencia presentada en el Coloquio de Estudios de Género: $A 25$ años de la fundación del PIEM. Ciudad de México: UNAM.

Scott, J. (1986). Gender: A useful category of historical analysis. The American Historical Review, 91(5), 1053-1075. https://doi.org/10.2307/1864376

SEP. (2013). Programa sectorial de educación (2013-2018). Recuperado de http://www.sep.gob.mx/

Tarrés, M. (octubre, 1996). Notas sobre los programas de estudios de género y de la mujer en el México de los noventa. Ponencia presentada en el Coloquio Anual de Estudios de Género en la UNAM. Ciudad de México: UNAM. https://doi.org/10.2307/j.ctv47w9dv.20

Taylor, S. y Bogdan, R (1987). Introducción a los métodos cualitativos de investigación: La búsqueda de significados. Barcelona: Paidós.

UNESCO. (2014a). Priority gender equality action plan (2014-2021). París: UNESCO.

UNESCO. (2014b). UNESCO's promise: Gender equality, a global priority. París: UNESCO.

Vázquez, V. y Zapata, E. (2005). Mujeres en universidades agronómicas y programas de estudios de la mujer en México y Estados Unidos: Un estudio comparativo. La Ventana. Revista de Estudios de Género, 21, 252-280. https://doi.org/10.18192/rceh.v42i1.1902

Verge, T., Ferrer-Fons, M. y González, M. (2017). Resistance to mainstreaming gender into the higher education curriculum. European Journal of Women's Studies, 25(1), 86-101. https://doi.org/10.1177/1350506816688237

Vélez, G. (s/f). La incorporación de la perspectiva de género en la Universidad Autónoma del Estado de México. Logros, retos y obstaculización. Recuperado de https://doi.org/10.18226/21789061.v10i3p441

West. L. (1999). The united nations women's conferences and feminist politics. En M. Meyer, y E. Prügl, (Eds.), Gender politics in global governance (pp. 177-195). Baltimore, MD: Rowman \& Little Field Publishers.

Yurén, T., Álvarez, A., Santamaría, D., Saenger, C. y Montes-de-Oca-O’Reilly, A. (2013). El espejismo de la equidad y las teorías implícitas en la relación TIC y género: El caso de estudiantes de una universidad pública estatal. En J. Espinosa (Coord.), El uso de las TIC en las universidades. Espejismos y disimulos (pp. 11-40). Ciudad de México: Ed. Miguel Ángel Porrúa. https://doi.org/10.2307/j.ctv11wjdp.7

\section{Breve CV de la autora}

\section{Alejandra Montes-de-Oca-O’Reilly}

Es Doctora en Humanidades por la Universidad de Texas. Realizó una estancia de investigación en la Universidad de Harvard. Llevó a cabo una estancia posdoctoral en el Instituto de Ciencias de la Educación de la Universidad Autónoma del Estado de Morelos (UAEM) durante casi dos años. Tiene reconocimiento como integrante del Sistema Nacional de Investigadores por parte del Consejo Nacional de Ciencia y Tecnología (CONACyT) en México. En 2015, recibió el Premio “Mujer Tec" en la Categoría "Investigación” por parte de Equality (red de 22 instituciones de educación superior de 21 
países de América Latina y Europa a favor de la equidad de género) y del Sistema Tecnológico de Monterrey. ORCID ID: http://orcid.org/0000-0001-6808-8182. Email: alemoreilly@gmail.com 